\title{
A ÁREA DE POLÍTICA, PLANEJAMENTO E GESTÃO EM SAÚDE NAS GRADUAÇÕES EM SAÚDE COLETIVA NO BRASIL
}

\author{
THE HEALTH POLICY, PLANNING, AND MANAGEMENT IN THE COLLECTIVE \\ HEALTH AREA IN UNDERGRADUATE EDUCATION IN BRAZIL
}

\author{
EL ÁREA DE POLÍTICA, PLANIFICACIÓN Y GESTIÓN EN SALUD EN \\ EL PREGRADO EN SALUD COLECTIVA EN BRASIL
}

\author{
Lorena Franco Sobral ${ }^{1}$ \\ Évelin Lúcia Barros ${ }^{2}$ \\ Leonardo Carnut ${ }^{3}$
}

Resumo Com a instrumentalização da área de política, planejamento e gestão em saúde, o ethos político do sanitarista vem sendo posto em questão em sua formação. Assim, buscou-se caracterizar os componentes curriculares relacionados com essa área nos cursos de graduação em saúde coletiva do Brasil. Em junho de 2014, fez-se um levantamento de dados secundários das matrizes curriculares pelo site do Ministério da Educação, cuja unidade de análise foi o componente curricular, usando-se o termo de referência para os cursos de graduação em saúde coletiva como parâmetro. Os cursos apresentam 'saúde coletiva' como principal denominação (60\%). A maior parte dos cursos se divide nas regiões Norte e Sudeste $(27 \%)$ e em instituições públicas (93\%). Sobre os conteúdos nas nomenclaturas, 'planejamento em saúde' é o mais citado (até sete vezes em uma mesma matriz) e em $60 \%$ do total dos cursos; $87 \%$ dos cursos não apresentam o termo 'Sistema Único de Saúde' como denominação de componente. Concluiu-se que sem uma padronização curricular, os futuros sanitaristas terão perfis diferenciados, atuando em uma perspectiva mais específica com tendência a um formato de gestão mais planificador e apresentando lacunas no que se refere aos conteúdos políticos e da defesa ideológica do Sistema Único de Saúde.

Palavras-chave políticas, planejamento e administração em saúde; educação superior; Sistema Único de Saúde; saúde pública; gestão em saúde.
Abstract With the instrumentalization of the health policy, planning, and management area, the public health physicians' political ethos has been being put into question in their training. Thus, we aimed to characterize the curricular components related to this area in undergraduate courses in collective health in Brazil. In June 2014, a secondary data survey of the curricular matrices was carried out on the Ministry of Education's website, the unit of analysis of which was the curricular component, using the reference term for undergraduate courses in collective health as a parameter. The courses present 'collective health' as the main denomination $(60 \%)$. Most of the courses are spread in the Northern and Southeastern regions (27\%) and in public institutions $(93 \%)$. On the contents in the nomenclatures, 'health planning' is the most cited (up to seven times in the same matrix) and in 60 percent of the total courses; 87 percent of the courses do not feature the term 'Unified Health System' as a component name. It was concluded that without a curricular standardization future public health physicians will have different profiles, acting in a more specific perspective leaning towards a more planning management format and with gaps regarding the political contents and the ideological defense of the Unified Health System.

Keywords policies, planning and administration in health; college education; Unified Health System; public health; management in health. 


\section{Introdução}

A saúde coletiva é um campo/núcleo de saberes e práticas de natureza interdisciplinar (Campos, 2000), que tem por objeto de estudo as 'necessidades sociais em saúde' (Paim e Almeida-Filho, 1998). A constituição desse campo é marcada pela ousadia na intercessão entre as áreas disciplinares para produzir um novo tipo de conhecimento que reúna a riqueza de suas partes (Leal e Camargo Júnior, 2012) na compreensão da saúde como processo socialmente determinado (Canesqui, 2007).

Esse campo científico se estrutura em um tripé com as seguintes características: utiliza uma ferramenta que desvenda a situação de saúde-doença-cuidado, seus riscos e determinantes de forma coletiva (a epidemiologia); vale-se da ação tecnológica, operada pelo aparelho do Estado, para a organização de serviços e sistemas de saúde em permanente debate com a sociedade civil (política, planejamento e gestão em saúde); e sua prática social se realiza sob a crítica reiterada ao modelo biomédico e com a busca por ações/serviços que operem maximizando a lógica da promoção da saúde (ciências sociais em saúde) (Associação Brasileira de Saúde Coletiva, 2013).

Dentre as áreas citadas, política, planejamento e gestão em saúde (PPGS) é a mais aplicada da saúde coletiva. Sem dúvida, é a área que demonstra a dupla dimensão da saúde coletiva (os saberes e as práticas) com maior clareza, orientando-se fortemente para a 'intervenção' (Paim e Teixeira, 2006), o que vem lhe promovendo grande racionalidade instrumental (Schraiber et al., 1999). Tal fato é justificado pela criação do Sistema Único de Saúde (SUS) como o evento histórico que permitiu a entrada expressiva do 'gerente' como novo profissional no setor público sanitário.

Assim, a noção de 'gerência em saúde' (Dussault, 1992) passou a guiar a representação social da práxis do campo. Segundo Schraiber e colaboradores (1999), admitiu-se naquele instante que a produção científica nessa área dificilmente seria baseada em uma teoria mais abstrata devido aos conflitos cotidianos da prática gerencial (técnicos/éticos/políticos) daqueles que se dedicavam a esse ofício.

Outra dificuldade adicional à investigação do campo é sua natureza polimorfa (Didonet, 1982). Em razão de congregar elementos teórico-conceituais de várias disciplinas, poucos são os pesquisadores que se sentem confortáveis em romper fronteiras disciplinares (Pátaro e Bovo, 2012), especialmente quando se agrega a difícil tarefa de relacionar diferentes paradigmas existentes na produção do conhecimento em cada área específica (Kuhn, 2003).

Logo, a PPGS tornou-se uma área que, em certos momentos, apresenta escassez de evidências que subsidiem a tomada de decisão, e quando estas existem, a agilidade para essa tomada desencoraja uma consulta mais aprofundada (Paim e Teixeira, 2006). Tradicionalmente, a pós-graduação em saúde coletiva vem se dedicando à tentativa de solucionar as lacunas das evidências científicas necessárias a essa área (Bosi e Paim, 2010). 
Entretanto, atualmente a discussão tem repousado na contribuição que as 'graduações' em saúde coletiva (Belisario et al., 2013; Castellanos et al., 2013) podem oferecer ao desenvolvimento da PPGS. Por ser a expressão indubitável da prática concreta do sanitarista, essa área merece cuidado especial na organização dos conteúdos essenciais para formação desse profissional e na definição dos temas que sejam prioridades para orientação de futuras pesquisas (Koifman e Gomes, 2008; Bosi e Paim, 2009).

Portanto, na ausência de estudos que se dediquem a essa tarefa, o estudo que deu origem a este artigo teve por objetivo caracterizar os componentes curriculares identificados nos cursos de graduação em saúde coletiva relacionados com a área de PPGS no Brasil. O intuito foi sistematizar a primeira contribuição para a compreensão sobre como a área se comporta na graduação.

\section{Contexto e caminho metodológico}

O estudo aqui apresentado foi parte do esforço de organização de pesquisadores de política, planejamento e gestão em saúde visando coletar dados sobre como se comporta a área nos cursos de graduação em saúde coletiva que estão sendo criados no país. Essa informação é estratégica para subsidiar o Plano Diretor da Comissão de Política, Planejamento e Gestão em Saúde da Associação Brasileira de Saúde Coletiva (Abrasco) no que concerne ao reordenamento da área. Consistiu o estudo em um levantamento de dados secundários (Vieira, 1980) - entendendo por dados secundários aqueles que são recolhidos (Mota e Carvalho, 2003) de um banco de dados preexistente. Trabalhou-se com o banco de dados referente aos cursos de graduação existentes no Brasil disponíveis no site do e-MEC do Ministério da Educação (<http://emec.mec.gov.br/>). Neste banco de dados, foram identificados os cursos de graduação de saúde coletiva existentes.

Em relação à identificação e à captação dos dados dos cursos de graduação em saúde coletiva, devido à não uniformidade dos termos relacionados ao título da profissão, utilizaram-se termos que se aproximam da área na busca pelo nome das graduações. Assim, foram alvo de busca as graduações em saúde coletiva (ou identidades congêneres com as seguintes sinonímias: 'saúde pública', 'gestão em saúde coletiva', 'gestão em saúde', 'saúde ambiental', dentre outras) visando captar todos aqueles cursos considerados de saúde coletiva e aqueles que, apesar de não estarem batizados com o nome de 'saúde coletiva', apresentam identidade com a área. No site do e-MEC, também foram coletados outros dados em relação a esses cursos, como: estados da federação aos quais pertencem, cidades, região, natureza institucional das universidades/faculdades que os ofertam (pública ou privada), assim como o site do curso para captação de suas respectivas matrizes curriculares, utilizando-as como documento fonte de informação para a lógica das análises documentais (Cellard, 2008). 
No que diz respeito à coleta das matrizes curriculares dos cursos, nessa etapa confeccionou-se um acervo de matrizes que foram analisadas segundo seus componentes curriculares. Entende-se por 'componente curricular' a soma de conhecimentos ou uma soma de disciplinas que ao final resultarão em um produto ou serviço. Para que esse produto ou serviço seja produzido, é pertinente ressaltar que ele se fará necessário à aplicação de conhecimentos (saber), habilidades (saber fazer) e atitudes (saber ser) constitutivos de uma competência; dessa forma, os 'componentes curriculares' estão associados à construção e à consolidação de uma competência específica (Ricardo, 2010) segundo as atribuições necessárias a uma profissão.

Como parâmetro de comparação para identificar os componentes curriculares associados à PPGS, utilizou-se o Termo de referência para os cursos de graduação em saúde coletiva (Associação Brasileira de Saúde Coletiva, 2013). $\mathrm{Na}$ época, as diretrizes curriculares nacionais (DCNs) para os cursos de graduação em saúde coletiva ainda estavam em construção, por isso recomendou-se que o documento que viabilizasse o amadurecimento da discussão sobre o assunto fosse o Termo de referência, para consolidação das futuras diretrizes curriculares para tais cursos. No âmbito da saúde coletiva, esse documento foi obtido como produto por meio do consenso técnico dos membros do Fórum de Graduação em Saúde Coletiva (FGSC) da Associação Brasileira de Saúde Coletiva, realizado em novembro de 2013 durante o VI Congresso Brasileiro de Ciências Sociais e Humanas em Saúde, na Universidade do Estado do Rio de Janeiro (Uerj). O fórum elencou os conteúdos mínimos que um curso de graduação em saúde coletiva deve ter em suas três principais áreas (eixo I: epidemiologia; eixo II: política, planejamento e gestão em saúde; eixo III: ciências sociais em saúde). O que se pretendeu, no estudo aqui apresentado, foi a identificação dos componentes curriculares que, por associação, possam conter os conteúdos que o Termo de referência apontava como essenciais para a área de política, planejamento e gestão em saúde, conforme o Quadro 1.

\section{Quadro 1}

\begin{tabular}{|ll|}
\hline Eixo II: política, planejamento e gestão em saúde & \multicolumn{1}{c|}{ Conteúdos } \\
\hline Área & Fundamentos de política contemporânea; fundamentos de gestão pública contemporânea; \\
& fundamentos de economia e financiamento em saúde; políticas sociais; política de saúde como uma \\
Gestão dos sistemas de saúde & política de proteção social; história das políticas de saúde no Brasil; sistemas de saúde; SUS; modelos \\
& de atenção à saúde; modelo de vigilância à saúde; financiamento e alocação de recursos em saúde; \\
& planejamento em saúde; sistema suplementar e planos e seguros privados de saúde. \\
\hline & Administração e gerência em saúde; território e saúde; informação em saúde; planejamento para \\
& unidades de saúde; gestão de materiais e medicamentos; gestão de pessoas em saúde; gestão de \\
& tecnologias em saúde; gestão financeira e orçamentária em saúde; gestão do trabalho em saúde; \\
& monitoramento e avaliação em saúde; regulação em saúde; auditoria em saúde.
\end{tabular}

Fonte: Termo de referência para os cursos de graduação em saúde coletiva (Associação Brasileira de Saúde Coletiva, 2013). 
O banco de dados foi organizado com base nas variáveis elencadas. Considerou-se cada conteúdo como uma variável do banco de dados, e sua presença ou ausência na matriz curricular foi identificada com uma codificação padrão. Quando em uma mesma matriz curricular houvesse vários componentes curriculares onde o conteúdo poderia estar previsto, registrou-se na variável o número de vezes que o conteúdo pôde estar disperso na matriz analisada.

Por fim, os dados coletados foram digitados no Excel for Windows versão 7.0. A análise dos dados ocorreu em uma fase descritiva. Nesta fase, foram feitas as distribuições de frequências e, quando apropriado, calculadas as medidas de tendência central, de dispersão e proporções.

No que se referiu às considerações éticas, trabalhou-se na pesquisa com dados secundários de uso público. Segundo a resolução n. 466/2012 CNS, para a manipulação de dados de domínio público não há necessidade de apreciação por um comitê de ética em pesquisa.

\section{Resultados}

Em consulta ao banco de dados no site do e-MEC, foram encontrados 18 cursos de graduação em saúde coletiva no Brasil ali registrados. Dos 18, 15 disponibilizavam suas grades curriculares em sítios eletrônicos na internet, isto é, as informações coletadas e apresentadas na pesquisa representaram $83,3 \%$ das graduações citadas antes. Em relação à nomenclatura adotada pelos cursos, as graduações com denominação saúde coletiva representaram 60\% (9) do total. Os outros nomes identificados foram saúde pública: $13 \%$ (2); gestão em saúde ambiental: 13\% (2); gestão em serviços de saúde: 7\% (1); e gestão em saúde: 7\% (1).

Quanto às regiões do Brasil, há mais graduações em saúde coletiva nas regiões Norte e Sudeste $(4 ; 27 \%)$ em comparação com as demais regiões. A maioria das graduações em saúde coletiva está sediada em instituições públicas (14; 93\%), e apenas uma (7\%) em instituição privada (Tabela 1). Nos componentes curriculares do eixo política, planejamento e gestão em saúde, 15 (100\%) dos cursos não apresentavam todos os conteúdos sobre PPGS existentes no Termo de referência.

\section{Tabela 1}

\begin{tabular}{lcc}
\hline Caracterização identitária, macrorregional e institucional dos cursos de graduação e saúde coletiva no Brasil - 2014 \\
\hline Variável & No & $\%$ \\
\hline Nomenclatura & 9 & $60 \%$ \\
Saúde coletiva & 2 & $13 \%$ \\
Saúde pública & 2 & $13 \%$ \\
Gestão em saúde ambiental & 1 & $7 \%$ \\
Gestão em serviços de saúde & 1 & $7 \%$ \\
Gestão em saúde & 15 & $100 \%$ \\
Total &
\end{tabular}




\section{Continuação - Tabela 1}

\begin{tabular}{lcc}
\hline Caracterização identitária, macrorregional e institucional dos cursos de graduação e saúde coletiva no Brasil - 2014 \\
\hline Variável & No & $\%$ \\
\hline Regiões & 4 & $27 \%$ \\
Norte & 3 & $20 \%$ \\
Nordeste & 3 & $20 \%$ \\
Sul & 4 & $27 \%$ \\
Sudeste & 1 & $6 \%$ \\
Centro-Oeste & 15 & 100 \\
Total & & \\
Natureza jurídica institucional & 14 & $93 \%$ \\
Pública & 1 & $7 \%$ \\
Privada & 15 & $100 \%$ \\
\hline
\end{tabular}

Fonte: Os autores.

A Tabela 2 explicita a caracterização dos cursos de graduação em saúde coletiva no Brasil segundo os conteúdos de gestão de sistemas de saúde presentes no Termo de referência (Associação Brasileira de Saúde Coletiva, 2013).

Tabela 2

\begin{tabular}{|c|c|c|c|c|c|c|c|c|c|c|}
\hline \multirow[t]{2}{*}{ Conteúdo de gestão de sistemas de saúde } & \multicolumn{10}{|c|}{ Número de citações nos componentes curriculares presentes nas matrizes estudadas } \\
\hline & Nenhuma & Uma & Duas & Três & Quatro & Cinco & Seis & Sete & Oito & Total \\
\hline Fundamentos da política contemporânea & $53 \%$ & $20 \%$ & $13 \%$ & $7 \%$ & $0 \%$ & $0 \%$ & $0 \%$ & $0 \%$ & $7 \%$ & $100 \%$ \\
\hline $\begin{array}{l}\text { Fundamentos de gestão pública } \\
\text { contemporânea }\end{array}$ & $67 \%$ & $33 \%$ & $0 \%$ & $0 \%$ & $0 \%$ & $0 \%$ & $0 \%$ & $0 \%$ & $0 \%$ & $100 \%$ \\
\hline $\begin{array}{l}\text { Fundamentos de economia e financiamento } \\
\text { em saúde }\end{array}$ & $67 \%$ & $20 \%$ & $6 \%$ & $7 \%$ & $0 \%$ & $0 \%$ & $0 \%$ & $0 \%$ & $0 \%$ & $100 \%$ \\
\hline Políticas sociais & $53 \%$ & $20 \%$ & $13 \%$ & $7 \%$ & $0 \%$ & $0 \%$ & $0 \%$ & $0 \%$ & $7 \%$ & $100 \%$ \\
\hline Políticas de saúde como políticas de proteção social & $20 \%$ & $46 \%$ & $7 \%$ & $7 \%$ & $13 \%$ & $0 \%$ & $7 \%$ & $0 \%$ & $0 \%$ & $100 \%$ \\
\hline História das políticas de saúde no Brasil & $73 \%$ & $20 \%$ & $0 \%$ & $7 \%$ & $0 \%$ & $0 \%$ & $0 \%$ & $0 \%$ & $0 \%$ & $100 \%$ \\
\hline Sistemas de saúde & $60 \%$ & $33 \%$ & $0 \%$ & $0 \%$ & $7 \%$ & $0 \%$ & $0 \%$ & $0 \%$ & $0 \%$ & $100 \%$ \\
\hline SUS & $87 \%$ & $6 \%$ & $7 \%$ & $0 \%$ & $0 \%$ & $0 \%$ & $0 \%$ & $0 \%$ & $0 \%$ & $100 \%$ \\
\hline Modelos de atenção à saúde & $40 \%$ & $27 \%$ & $27 \%$ & $0 \%$ & $6 \%$ & $0 \%$ & $0 \%$ & $0 \%$ & $0 \%$ & $100 \%$ \\
\hline Modelos de vigilância à saúde & $20 \%$ & $27 \%$ & $20 \%$ & $6 \%$ & $20 \%$ & $7 \%$ & $0 \%$ & $0 \%$ & $0 \%$ & $100 \%$ \\
\hline Financiamento e alocação de recursos em saúde & $60 \%$ & $33 \%$ & $7 \%$ & $0 \%$ & $0 \%$ & $0 \%$ & $0 \%$ & $0 \%$ & $0 \%$ & $100 \%$ \\
\hline Planejamento em saúde & $0 \%$ & $60 \%$ & $6 \%$ & $7 \%$ & $0 \%$ & $13 \%$ & $7 \%$ & $0 \%$ & $7 \%$ & $100 \%$ \\
\hline $\begin{array}{l}\text { Sistemas suplementares e planos e seguros } \\
\text { privados em saúde }\end{array}$ & $67 \%$ & $33 \%$ & $0 \%$ & $0 \%$ & $0 \%$ & $0 \%$ & $0 \%$ & $0 \%$ & $0 \%$ & $100 \%$ \\
\hline
\end{tabular}

Fonte: Os autores. 
Dentre os conteúdos essenciais presentes no Termo de referência para a área de política, planejamento e gestão em saúde, foram identificados seus percentuais de ausência e presença nas matrizes curriculares dos cursos de graduação em saúde coletiva estudados conforme comparação com o Quadro 1. Identificou-se a ausência dos conteúdos sobre 'fundamentos de política contemporânea' em 53\% (8) das matrizes curriculares; 'fundamentos da gestão pública contemporânea' em 67\% (10); e 'fundamentos de economia e financiamento em saúde' em 67\% (10). Sobre 'fundamentos de economia e financiamento em saúde', 33\% das matrizes curriculares citaram este conteúdo pelo menos uma vez; nas vezes em que foi citado, veio identificado nas seguintes porcentagens: citado uma vez $-20 \%$ (3); duas vezes $-6 \%$ (1); e três vezes $-7 \%$ (1).

Conteúdos de 'políticas sociais' não constam nas matrizes curriculares de 53\% (8) dos cursos de graduação em saúde coletiva. Quando esses conteúdos se fazem presentes, eles são citados pelo menos uma vez em $20 \%$ (3), duas vezes em $13 \%$ (2), três vezes em 7\% (1) e oito vezes em 7\% (1). Já as 'políticas de saúde como políticas de proteção social' foram explicitadas pelo menos uma vez nos currículos em $46 \%$ (7) dos cursos, variando em $20 \%$ (3): nenhuma vez; $13 \%$ (2): quatro vezes; e $7 \%$ (1): seis vezes.

Em 73\% (11) das matrizes curriculares pesquisadas, não foram identificados componentes curriculares de 'história das políticas de saúde no Brasil' ou assemelhados. Nos $27 \%$ dos cursos em que esse conteúdo surgiu, ele foi citado pelo menos uma vez $(20 \%$; 3$)$ e três vezes $(7 \% ; 1)$. Em $60 \%$ das graduações, não havia um componente curricular de 'sistemas de saúde'. Quando este conteúdo foi identificado, 33\% das matrizes o mencionaram pelo menos uma vez e 7\% quatro vezes. Oitenta e sete por cento (13) dos cursos não apresentaram disciplinas relacionadas ao termo 'Sistema Único de Saúde'. Dos que apresentaram, apenas $6 \%$ o citaram pelo menos uma vez e $7 \%$ duas vezes, sendo esse conteúdo o menos identificado. Conteúdos relacionados aos 'modelos de atenção à saúde' não foram identificados em $40 \%$ das matrizes pesquisadas. Quando esse termo surgiu, foi citado uma vez $(27 \%)$, duas vezes $(27 \%)$ e quatro vezes $(6 \%)$.

Componentes curriculares com denominação 'modelos de vigilância à saúde' ou semelhantes foram citados pelo menos uma vez $(27 \% ; 4)$, duas vezes $(20 \% ; 3)$, três vezes $(6 \% ; 1)$, quatro vezes $(20 \% ; 3)$, cinco vezes $(7 \% ; 1)$ e nenhuma vez $(20 \%$; 3$)$. A respeito da denominação 'financiamento e alocação de recursos em saúde", $60 \%$ das matrizes não apresentaram este tema; dos $40 \%$ que tinham esse conteúdo, $29 \%$ o citaram apenas uma vez e $7 \%$, duas vezes.

Sobre o conteúdo 'planejamento em saúde', 60\% das graduações o apresentaram pelo menos uma vez em suas grades curriculares. Outras matrizes curriculares o mencionaram em diferentes proporções: $6 \%$ duas vezes, $7 \%$ três vezes, $13 \%$ cinco vezes, $7 \%$ seis vezes e $7 \%$ oito vezes, sendo esse conteúdo um dos mais citados nas matrizes estudadas. 
Em $67 \%$ das matrizes, não foram identificados conteúdos referentes a 'sistemas suplementares e planos e seguros privados em saúde'. Das matrizes que o mencionaram, 33\% o citaram uma vez apenas.

Os conteúdos referentes à área de gerência de serviços de saúde foram caracterizados de acordo com a Tabela 3.

Tabela 3

\begin{tabular}{|c|c|c|c|c|c|c|c|c|c|c|}
\hline \multirow[t]{2}{*}{ Conteúdo de gestão de sistemas de saúde } & \multicolumn{10}{|c|}{ Número de citações nos componentes curriculares presentes nas matrizes estudadas } \\
\hline & Nenhuma & Uma & Duas & Três & Quatro & Cinco & Seis & Sete & Oito & Total \\
\hline Administração e gerência em saúde & $20 \%$ & $6 \%$ & $27 \%$ & $13 \%$ & $20 \%$ & $0 \%$ & $7 \%$ & $0 \%$ & $7 \%$ & $100 \%$ \\
\hline Território e saúde & $73 \%$ & $13 \%$ & $7 \%$ & $0 \%$ & $0 \%$ & $0 \%$ & $0 \%$ & $7 \%$ & $0 \%$ & $100 \%$ \\
\hline Informação em saúde & $46 \%$ & $27 \%$ & $20 \%$ & $0 \%$ & $0 \%$ & $0 \%$ & $7 \%$ & $0 \%$ & $0 \%$ & $100 \%$ \\
\hline Planejamento para unidades de saúde & $93 \%$ & $7 \%$ & $0 \%$ & $0 \%$ & $0 \%$ & $0 \%$ & $0 \%$ & $0 \%$ & $0 \%$ & $100 \%$ \\
\hline Gestão de materiais e medicamentos em saúde & $73 \%$ & $27 \%$ & $0 \%$ & $0 \%$ & $0 \%$ & $0 \%$ & $0 \%$ & $0 \%$ & $0 \%$ & $100 \%$ \\
\hline Gestão de pessoas em saúde & $60 \%$ & $27 \%$ & $6 \%$ & $0 \%$ & $7 \%$ & $0 \%$ & $0 \%$ & $0 \%$ & $0 \%$ & $100 \%$ \\
\hline Gestão de tecnologias em saúde & $67 \%$ & $6 \%$ & $13 \%$ & $7 \%$ & $7 \%$ & $0 \%$ & $0 \%$ & $0 \%$ & $0 \%$ & $100 \%$ \\
\hline Gestão financeira e orçamentária em saúde & $53 \%$ & $40 \%$ & $7 \%$ & $0 \%$ & $0 \%$ & $0 \%$ & $0 \%$ & $0 \%$ & $0 \%$ & $100 \%$ \\
\hline Monitoramento e avaliação em saúde & $27 \%$ & $53 \%$ & $6 \%$ & $7 \%$ & $0 \%$ & $7 \%$ & $0 \%$ & $0 \%$ & $0 \%$ & $100 \%$ \\
\hline Regulação em saúde & $80 \%$ & $20 \%$ & $0 \%$ & $0 \%$ & $0 \%$ & $0 \%$ & $0 \%$ & $0 \%$ & $0 \%$ & $100 \%$ \\
\hline Auditoria em saúde & $80 \%$ & $13 \%$ & $7 \%$ & $0 \%$ & $0 \%$ & $0 \%$ & $0 \%$ & $0 \%$ & $0 \%$ & $100 \%$ \\
\hline
\end{tabular}

'Administração e gerência em saúde' é um conteúdo que 80\% das matrizes apresentaram em sua constituição, citando-o pelo menos uma vez $(6 \% ; 1)$, duas vezes $(27 \% ; 4)$, três $(13 \% ; 2)$, quatro vezes $(20 \% ; 3)$, seis $(7 \% ; 1)$ e oito vezes $(7 \% ; 1)$. Sobre o conteúdo relacionado a 'território e saúde', 73\% (11) das matrizes curriculares não citaram esses conteúdos em sua composição; em $27 \%$ das vezes em que se mencionou esse conteúdo, ele foi identificado nas seguintes porcentagens: uma vez $(13 \% ; 2)$, duas vezes $(7 \% ; 1)$ e sete vezes $(7 \% ; 1)$. Quanto aos conteúdos sobre 'informação em saúde', 46\% (7) não foram citados nas matrizes curriculares; em $54 \%$ das vezes em que o conteúdo foi mencionado, identificou-se nas seguintes proporções: pelo menos uma vez: $27 \%$; duas vezes: $20 \%$; e seis vezes: $7 \%$.

Para o termo 'planejamento para unidades de saúde', não se identificaram conteúdos relacionados em 93\% (14) das matrizes curriculares, sendo citado apenas uma vez $(7 \%)$. Conteúdos relacionados ao componente 'gestão de materiais e medicamentos (insumos) em saúde' não foram 
mencionados em 73\% (11) das matrizes curriculares; apenas $27 \%$ (4) o citaram uma vez.

Em $60 \%$ (9) das matrizes curriculares estudadas, não foram identificados conteúdos sobre o componente curricular 'gestão de pessoas em saúde'; sobre as que apresentaram, as proporções foram as seguintes: pelo menos uma vez $(27 \% ; 4)$, duas vezes $(6 \% ; 1)$ e quatro vezes $(7 \% ; 1)$. Conteúdos sobre 'gestão de tecnologias em saúde' não foram identificados em 67\% (10) das matrizes curriculares pesquisadas; nos $33 \%$ das vezes em que foi citado, variou nas seguintes porcentagens: uma vez $(6 \%)$, duas vezes $(13 \%)$, três vezes $(7 \%)$ e quatro vezes $(7 \%)$.

Não foram explicitados conteúdos com nomenclatura 'gestão financeira e orçamentária em saúde' ou semelhante em 53\% (8) das matrizes curriculares. Quando eles existem, são citados pelo menos uma vez $(40 \% ; 6)$ e duas vezes $(7 \% ; 1)$. Quanto ao componente curricular 'monitoramento e avaliação em saúde', $27 \%$ (4) das graduações em saúde coletiva não apresentaram esse conteúdo. Das $73 \%$ (11) que o citaram, as proporções variaram: uma vez (53\%; 8), duas vezes $(6 \% ; 1)$, três vezes $(7 \% ; 1)$ e cinco vezes $(7 \% ; 1)$. Em $80 \%$ (12) das matrizes curriculares estudadas, não se identificaram em sua composição conteúdos relacionados à 'regulação em saúde'. Dos $20 \%$ (3) restantes, foi mencionado pelo menos uma vez. Termos com denominação 'auditoria em saúde' ou semelhante não apresentaram conteúdos em $80 \%$ (12) das matrizes curriculares, tendo sido identificados pelo menos uma vez $(13 \% ; 2)$ e duas vezes $(7 \% ; 1)$.

\section{(Re)pensando a área de política, planejamento e gestão em saúde nas graduações em saúde coletiva}

A pesquisa aqui apresentada caracterizou a área de política, planejamento e gestão em saúde como área do conhecimento sanitário nos cursos de graduação em saúde coletiva no Brasil. A ideia principal foi conhecer a dedicação dos cursos a essa área para fomentar um debate sobre padronização mínima que garanta identidade laboral àqueles estudantes egressos desses cursos.

No que tange à sua caracterização identitária, tais graduações são chamadas, em sua maioria, de 'saúde coletiva'; no entanto, foram constatadas outras sinonímias, significando a não padronização quanto à terminologia mais apropriada para esses cursos no país. Segundo Campos (2000), o termo 'saúde coletiva' é o que melhor caracteriza o campo de atuação do sanitarista. Definida como um campo/núcleo de saberes e práticas, a saúde coletiva considera a saúde como um produto social e, como afirma Nunes (1996), sua prática na gestão dos sistemas/serviços visa, em última instância, superar a medicalização e o contraste público-privado ressaltando a temática em seu caráter coletivo.

A distribuição macrorregional se apresentou de forma desigual perante as cinco macrorregiões do país. As graduações mostraram-se concentradas nas 
regiões Norte e Sudeste, seguidas pelas demais. É interessante dar ênfase ao fato de que o primeiro curso de graduação em saúde coletiva foi implantado na região Sul (Universidade Federal do Rio Grande do Sul). Uma das hipóteses para os números de graduações em saúde coletiva serem maiores nessas duas regiões específicas é que a primeira (Norte) apresenta particularidades regionais que justificam o investimento na formação de novos recursos humanos em saúde para lidar com o problema da forte disparidade regional que assola a região do ponto de vista formativo. Gil (2005) já expressava tal situação para a formação de profissionais para o exercício da prática assistencial na área de saúde da família, o que sugere que a região Norte pode estar sendo tradicionalmente desassistida nesse sentido.

A região Sudeste apresentou-se em segundo lugar do ponto de vista de concentração de cursos de graduação em saúde coletiva. Uma hipótese para tal situação é que a região é a mais desenvolvida do país e, correlatamente, o espaço tradicional de desenvolvimento do campo de trabalho no âmbito da saúde coletiva (com a notável exceção da Bahia), que sempre esteve concentrado no eixo Rio-São Paulo (Castro-Santos, 1993).

Dos 15 cursos de saúde coletiva, apenas um pertencia a uma instituição privada, sugerindo que a imprevisibilidade de inserção profissional no mercado promove uma baixa procura pelo curso, desencorajando as instituições privadas a tomá-la como modelo de negócio. É possível pensar que a profissão apresenta dificuldades de conformação aos novos moldes da reestruturação produtiva (Catani, Oliveira e Dourado, 2001), o que lhe fornece menos prestígio do ponto de vista mercadológico. Por outro lado, a concentração dos cursos em instituições públicas pode indicar que o corpo docente ousa mais quando há esse interesse. A autonomia de que essas instituições gozam ajuda na criação de cursos que ainda não têm reconhecimento, como também assegura a continuidade independentemente do número de matriculados. Nessa perspectiva, espera-se mais liberdade à crítica ao modelo biomédico (Tavares, 1997), tão necessária a uma prática gerencial coerente com o que se vislumbra no horizonte teórico da saúde coletiva no Brasil (Campos, 2000).

No que concerne à caracterização dos conteúdos referentes à gestão de sistemas de saúde, pôde-se identificar nas matrizes curriculares a ausência de conteúdos sobre 'fundamentos de política contemporânea', 'fundamentos da gestão pública contemporânea' e 'fundamentos de economia e financiamento em saúde'. A inexistência desses conteúdos nos cursos de graduação em saúde coletiva pode ser considerada um problema, pois a compreensão da categoria política em sua polissemia e sua intrínseca relação com o poder econômico (Pio e Porto, 1998) e com a administração estatal (em suas múltiplas vertentes) (Secchi, 2009) são cruciais para a construção do conhecimento nessa área.

Em relação aos conteúdos de 'políticas sociais', 53\% dos cursos não disponibilizavam em suas matrizes curriculares esse componente. No contexto 
prático, o estudante de saúde coletiva será um operador de uma política social como é considerada a política de saúde no Brasil (Fleury e Ouverney, 2012). Parece estranho, mas nas matrizes observadas não houve nenhuma referência explícita a esse conteúdo, e para que o direito à saúde se concretize, outros direitos sociais operacionalizados pelas demais políticas sociais (Simões, 2013) devem ser minimamente amparados, o que justifica essa pauta na formação.

No que se refere aos conteúdos de 'história de políticas de saúde no Brasil', 73\% das graduações não a explicitaram em suas matrizes. É possível supor que esse conteúdo possa (deva) ser trabalhado em outras disciplinas com diversas nomenclaturas, destinando a esse conteúdo um lugar de tópico ou capítulo a ser estudado dentro de um macrotema. Mesmo sendo um consenso a importância desse conteúdo (Paim, 2006) para a compreensão do contexto sócio-histórico da criação do SUS, sua invisibilidade nas matrizes pode sugerir certa desimportância.

O tema 'sistemas de saúde e Sistema Único de Saúde' também goza de uma invisibilidade que, em se tratando da extrema centralidade do tema na formação, merece destaque. Para Foucault (1999), as palavras são dotadas de um poder inerente que enfraquece ou reaviva um contexto semântico. Crê-se, portanto, que a invisibilidade do termo 'Sistema Único de Saúde' em uma matriz de um curso de formação de sanitaristas não é por acaso e pode estar relacionada com a perda da força ideológica originária que o SUS deteve no período da Reforma Sanitária brasileira (Paim, 2006).

Em 40\% das matrizes, os conteúdos sobre 'modelos de atenção à saúde' não existiam ou encontravam-se também invisibilizados. Supõe-se que tópicos essenciais como rede, regionalização, hierarquização dos serviços de saúde e a própria ideia de modelo como essencial na imaginação sanitária (racionalidade) (Paim, 2012) sobre o sistema possam estar comprometidos. Já em 'financiamento e alocação de recursos' registrou-se o percentual de $60 \%$ de ausência nas matrizes pesquisadas. Marques e Mendes (2007) ressaltam o quanto o financiamento e a alocação de recursos em saúde no Brasil são complexos e explicitam sua essencialidade na formação em políticas sociais.

Preenchendo esse 'vazio conteudístico', segue-se o 'planejamento em saúde', que contabilizou até $60 \%$ de presença nas matrizes. Mesmo ao se reconhecer a importância do planejamento como conteúdo essencial para o sanitarista, é possível afirmar que a maior parte das matrizes foca seus conteúdos nesse tema específico. Pode ser que, pelas contingências do âmbito da política/economia, os formuladores das matrizes esperam formar mais um 'burocrata' à luz da 'neutralidade' provida pela função de administrador público (Behn, 1998) do que necessariamente um profissional capaz de fazer a mudança social por dentro do aparelho do Estado (Sell, 2006). Este argumento pode ser reforçado quando se vê que o tema 'sistemas suplementares e planos e seguros privados em saúde' não foi identificado em $67 \%$ das matrizes estudadas. 
Com relação à caracterização dos conteúdos referentes à gerência de serviços de saúde, 'administração e gerência em saúde' foi citado em grande parte das matrizes curriculares. Espera-se que nessa área o foco administrativo prevaleça, já que a ação mais objetiva da atuação do sanitarista é a administração dos serviços (Campos, 2000).

'Território e saúde' não foi citado em 73\% das matrizes curriculares pesquisadas. Apesar de poder constar como um conteúdo em componente com outra denominação, sua ausência explícita tornou questionável a intervenção sanitária dos formandos dessas matrizes, já que o território deve ser considerado como tecido vivo (Santos, 1997) e, portanto, espaço de ação concreta desse profissional. Parece coerente essa argumentação quando se examina o tema 'informação em saúde', que nem sempre apareceu explícito nas matrizes, sugerindo uma desimportância da informação em saúde para o reconhecimento da realidade da população e como insumo do planejamento (Mota e Carvalho, 2003) de uma intervenção em um território específico.

Alguns conteúdos que trabalham a prática sanitária em âmbito local parecem desimportantes também. Este é o caso de 'gestão de material de medicamentos e insumos', não citado em $73 \%$ das matrizes. 'Gestão de pessoas em saúde' não foi citado em $60 \%$ das matrizes; 'gestão de tecnologias em saúde', em $67 \%$; 'gestão financeira e orçamentária em saúde', em 53\%; 'regulação em saúde' e 'auditoria em saúde', em $80 \%$. Especialmente esse núcleo de conhecimentos mais orientados à prática sanitária em âmbito local (tanto na gestão do cotidiano dos serviços de assistência quanto na prática gerencial local em distritos sanitários ou secretarias municipais) parece não gozar de peso substantivo na formulação de componentes curriculares na área de política, planejamento e gestão nos cursos estudados, devido a sua alta proporção de ausência em bloco. O pensamento gerencial em saúde, justificador da existência da profissão especialmente nos anos 1970-1980 (Schraiber, 1999), com as primeiras iniciativas do ensino de gerência de serviços de saúde - a exemplo do Projeto de Desenvolvimento Gerencial de Unidades de Saúde do SUS (Gerus) (Brasil, 2017) -, caiu em desuso, sugerindo a dispensabilidade desses conteúdos conforme os dados apresentados. É pertinente notar que a prática gerencial local, tão essencial no desenvolvimento da rede de atenção primária e responsável pela consolidação da municipalização, parece não ser um projeto vislumbrado por esses currículos. É possível que os futuros profissionais graduados neste país não detenham as competências necessárias para completar a municipalização dentro das suas possibilidades - mesmo sob o lema da 'regionalização' como 'o caminho' (Santos e Andrade, 2013) - devido à ausência de tais temas. Assim, esse achado sugere que os cursos aqui analisados poderiam ser responsabilizados por ensejarem um descuido dessa atribuição do sanitarista em construir um sistema de saúde ascendente e fincado em bases sólidas (considerando o município como unidade jurídico-política fundamental para esse propósito). 


\section{Considerações finais}

Identificaram-se 18 instituições de ensino superior que ofertavam cursos de graduação em saúde coletiva no Brasil no período da pesquisa. Destas, apenas 15 disponibilizavam suas matrizes curriculares, que foram coletadas no banco de dados e-MEC. Na análise quantitativa dos componentes curriculares nas matrizes relacionadas à área de política, planejamento e gestão em saúde, constatou-se que esses componentes estavam caracterizados seguindo uma linha de organização com o objetivo de formar graduandos com um perfil mais planejador e administrativo, com foco em nível central. Mesmo com as atuais diretrizes curriculares nacionais para os cursos de graduação em saúde coletiva, recém-editadas em junho de 2015 pelo Fórum de Graduação em Saúde Coletiva no país, a realização da pesquisa que deu origem a este artigo procurou justificar ainda mais o papel das novas diretrizes em rever suas opções normativas, pois aqueles que formulam as matrizes curriculares dos cursos de graduação em saúde coletiva no Brasil parecem não contemplar parte fundamental da área de PPGS necessária à formação.

Mesmo com essas limitações, foi possível perceber que quem organiza as matrizes segue uma lógica que tenta responder às particularidades regionais em que o curso está inserido. Contudo, alerta-se que sem uma padronização curricular, que defina conteúdos mínimos na construção dessas matrizes, é possível que se perpetue a formação de futuros sanitaristas tal qual o perfil apresentado no estudo: um sanitarista sem homogeneidade, com perspectiva regional mais específica, de tendência a um formato de gestão mais planificador e com lacunas no que se refere aos conteúdos políticos e da defesa ideológica do SUS.

\section{Colaboradores}

Todos os autores participaram igualmente, desde a concepção da ideia até a execução do manuscrito. Declaramos que não há conflitos de interesse. 
Resumen Con la instrumentalización del área política, planificación y gestión en salud, el ethos político del profesional de la salud está sobre la mesa desde su formación. Así, se buscó caracterizar los componentes curriculares relacionados con esta área en los cursos de pregrado en salud colectiva en Brasil. Se realizó un relevamiento de datos secundarios en junio de 2014 de las matrices curriculares a través del sitio del Ministerio de Educación, cuya unidad de análisis fue el componente curricular, siendo utilizado el término de referencia para los cursos de pregrado en salud colectiva como parámetro. Los cursos presentan 'salud colectiva' como principal denominación (60\%). La mayor parte de los cursos se encuentra en las regiones Norte y Sudeste $(27 \%)$ y en instituciones públicas $(93 \%)$. Sobre los contenidos en las nomenclaturas, "planificación en salud" es el más citado (hasta siete veces en una misma matriz) y en el $60 \%$ del total de los cursos; el $87 \%$ de los cursos no presentan el término 'Sistema Único de Salud' como denominación de componente. Se concluyó que sin una normalización curricular, los futuros profesionales de la salud tendrán perfiles diferenciados, actuando en una perspectiva más específica con tendencia a un formato de gestión más planificador y presentando vacíos en lo que se refiere a los contenidos políticos y de la defensa ideológica del Sistema Único de Salud.

Palabras clave políticas, planificación y administración en salud; educación superior; Sistema Único de Salud; salud pública; gestión en salud.

\section{Notas}

${ }^{1}$ Universidade de Pernambuco, Faculdade de Ciências Médicas, Santo Amaro, Recife, Pernambuco, Brasil.

$<$ lorenfsol@hotmail.com>

Correspondência: Avenida Doutor Cláudio José Gueiros Leite, 1.022, Janga, CEP 53437-000, Paulista, Pernambuco, Brasil.

${ }^{2}$ Universidade de Pernambuco, Faculdade de Ciências Médicas, Santo Amaro, Recife, Pernambuco, Brasil.

<evinhabarros1@hotmail.com>

${ }^{3}$ Universidade de Pernambuco, Faculdade de Ciências Médicas, Santo Amaro, Recife, Pernambuco, Brasil.

$<$ leonardo.carnut@upe.br>

\section{Referências}

\begin{abstract}
ASSOCIAÇÃO BRASILEIRA DE SAÚDE COLETIVA (ABRASCO). Fórum de Graduação em Saúde Coletiva (FGSC). Termo de referência para os cursos de graduação em saúde coletiva. Rio de Janeiro: Abrasco, 2013. Disponível em: <https://docs.google.com/document/d/1IW_ lrxTLiKm94Z5PaYBtLTUxdAMe8N2OiAjtNjNYbWs/edit>. Acesso em: 21 nov. 2013.
\end{abstract}

BEHN, Robert D. O novo paradigma da gestão pública e a busca pela accountability demo- crática. Revista do Serviço Público, Brasília, v. 49, n. 4, p. 5-43, 1998.

BELISARIO, Soraya A. et al. Implantação do curso de graduação em saúde coletiva: a visão dos coordenadores. Ciência \& Saúde Coletiva, Rio de Janeiro, v. 18, n. 6, p. 1.625-1.634, 2013.

BOSI, Maria L. M.; PAIM, Jairnilson S. Graduação em saúde coletiva: subsídios para um debate necessário. Cadernos de Saúde Pública, Rio de Janeiro, v. 25, n. 2, p. 236-237, 2009. 
BOSI, Maria L. M.; PAIM, Jairnilson S. Graduação em saúde coletiva: limites e possibilidades como estratégia de formação profissional. Ciência \& Saúde Coletiva, Rio de Janeiro, v. 15, n. 4 , p. $2.029-2.038,2010$.

BRASIL. Ministério da Saúde. Fundação Oswaldo Cruz. Escola Nacional de Saúde Pública Sergio Arouca. Projeto de Desenvolvimento Gerencial de Unidades Básicas do Sistema Único de Saúde. Disponível em: <http://www.ensp.fiocruz.br/ portal-ensp/escola-de-governo/cursos/index. php? idcurso $=325 \&$ ind tipo $=2 \&$ menu $=661>$. Acesso em: 25 jun. 2017.

CAMPOS, Gastão W. S. Saúde pública e saúde coletiva: campo e núcleo de saberes e práticas. Ciência \& Saúde Coletiva, Rio de Janeiro, v. 5, n. 2, p. 219-230, 2000.

CANESQUI, Ana M. Ciências sociais e saúde no Brasil. São Paulo: Hucitec/Aderaldo \& Rothschild, 2007.

CASTELLANOS, Marcelo E. P. et al. Estudantes de graduação em saúde coletiva: perfil sociodemográfico e motivações. Ciência \& Saúde Coletiva, Rio de Janeiro, v. 18, n. 6, p. 1.657-1.666, 2013.

CASTRO-SANTOS, Luiz A. A Reforma Sanitária pelo alto: o pioneirismo paulista no início do século XX. Dados: Revista de Ciências Sociais, Rio de Janeiro, v. 36, n. 3, p. 361-392, 1993.

CATANI, Afrânio M.; OLIVEIRA, João F.; DOURADO, Luiz F. Política educacional, mudanças no mundo do trabalho e reforma curricular dos cursos de graduação no Brasil. Educação \& Sociedade, Campinas, ano XXII, n. 75, p. 67-83, 2001.

CELLARD, André. Análise documental. In: POUPART, Jean et al. A pesquisa qualitativa: enfoques epistemológicos e metodológicos. Petrópolis: Vozes, 2008. p. 295-316.

DIDONET, Leónidas M. Ensaio de interdisciplinaridade. Revista Portuguesa de Filosofia, Braga, v. 38, n. 4, p. 305-316, 1982.

DUSSAULT, Gilles. Gestão dos serviços públicos de saúde: características e exigências. Revista de Administração Pública, Rio de Janeiro, v. 26, n. 2, p. 8-19, 1992.

FLEURY, Sônia; OUVERNEY, Assis M. Politica de saúde: uma política social. In: GIOVANELLA, Lígia; ESCOREL, Sarah (orgs.). Politicas e sistemas de saúde no Brasil. 2. ed. Rio de Janeiro: Editora Fiocruz, 2012. p. 25-58.

FOUCAULT, Michel. As palavras e as coisas: uma arqueologia das ciências humanas. 8. ed. São Paulo: Martins Fontes, 1999.

GIL, Célia R. R. Formação de recursos humanos em saúde da família: paradoxos e perspectivas. Cadernos de Saúde Pública, Rio de Janeiro, v. 21, n. 2, p. 490-498, 2005.

KOIFMAN, Lilian; GOMES, Lina N. A graduação em saúde coletiva: um debate ou uma realidade? Revista Brasileira de Educação Médica, Rio de Janeiro, v. 32, n. 4, p. 417-418, 2008.

KUHN, Thomas. A estrutura das revoluções cientificas. 7. ed. São Paulo: Perspectiva, 2003.

LEAL, Mariana B.; CAMARGO JÚNIOR, Kenneth R. Saúde coletiva em debate: reflexões acerca de um campo em construção. Interface: Comunicação, Saúde e Educação, Botucatu, v. 16, n. 40, p. 53-66, 2012.

MARQUES, Rosa M.; MENDES, Áquilas. Servindo a dois senhores: as políticas sociais no governo Lula. Revista Katálysis, Florianópolis, v. 10, n. 1, p. 15-23, 2007.

MOTA, Eduardo; CARVALHO, Déa M. Sistemas de informação em saúde. In: ROUQUAYROL, Maria Z. Epidemiologia e saúde. 6. ed. Rio de Janeiro: Medsi, 2003. p. 605-628.

NUNES, Everardo D. Saúde coletiva: revisitando a sua história e os cursos de pós-graduação. Ciência \& Saúde Coletiva, Rio de Janeiro, v. 1, n. 1, p. 55-69, 1996.

PAIM, Jairnilson S. Planejamento em saúde para não especialistas. In: CAMPOS, Gastão W. S. et al. Tratado de saúde coletiva. São Paulo: Hucitec; Rio de Janeiro: Editora Fiocruz, 2006. p. 767-782. 
PAIM, Jairnilson S. Modelos de atenção à saúde. In: GIOVANELLA, Lígia; ESCOREL, Sarah (orgs.). Politicas e sistemas de saúde no Brasil. 2. ed. Rio de Janeiro: Editora Fiocruz, 2012. p. 459-491.

PAIM, Jairnilson S.; ALMEIDA-FILHO, Naomar. Saúde coletiva: uma 'nova saúde pública' ou campo aberto a novos paradigmas? Revista de Saúde Pública, São Paulo, v. 32, n. 4, p. 299-316, 1998.

PAIM, Jairnilson S.; TEIXEIRA, Carmem F. Política, planejamento e gestão em saúde: um balanço do estado da arte. Revista de Saúde Pública, São Paulo, v. 40, n. esp., p. 8-73, 2006.

PÁTARO, Ricardo F.; BOVO, Marcos C. A interdisciplinaridade como possibilidade de diálogo e trabalho coletivo no campo da pesquisa e da educação. Revista Nupem, Campo Mourão, v. 4, n. 6, p. 46-63, 2012.

PIO, Carlos; PORTO, Mauro. Teoria política contemporânea: política e economia segundo os argumentos elitistas, pluralistas e marxistas. In: RUA, Maria G.; CARVALHO, Maria I. V. (orgs.). O estudo da política: tópicos selecionados. Brasília: Paralelo 15, 1998. p. 291-314.

RICARDO, Elio C. Discussão acerca do ensino por competências: problemas e alternativas. Cadernos de Pesquisa, São Paulo, v. 40, n. 140, p. 605-628, 2010.

SANTOS, Lenir; ANDRADE, Luiz O. M. Rede interfederativa de saúde. In: SILVA, Silvio F. (org.). Redes de atenção à saúde: desafios da regio- nalização do SUS. 2. ed. Campinas: Saberes Editora, 2013. p. 35-74.

SANTOS, Milton. A natureza do espaço: técnica e tempo - espaço e emoção. 2. ed. São Paulo: Hucitec, 1997.

SCHRAIBER, Lilia B. et al. Planejamento, gestão e avaliação em saúde: identificando problemas. Ciência \& Saúde Coletiva, Rio de Janeiro, v. 4, n. 2, p. 221-242, 1999.

SECCHI, Leonardo. Modelos organizacionais e reformas da administração pública. Revista de Administração Pública, Rio de Janeiro, v. 43, n. 2, p. 347-369, 2009.

SELL, Carlos E. Democracia. Introdução à sociologia política: política e sociedade na modernidade tardia. Rio de Janeiro: Vozes, 2006. p. 79-108.

SIMÕES, Carlos. Os direitos sociais na Constituição de 1988: conceito. In: SIMÕES, Carlos. Teoria e crítica dos direitos sociais: o Estado social e o estado democrático de direito. São Paulo: Cortez, 2013. p. 171-201.

TAVARES, Clotilde. A matriz invisível da saúde. In: TAVARES, Clotilde. Iniciação à visão holística. 3. ed. Rio de Janeiro: Record, 1997. p. 76-92.

VIEIRA, Sônia. Introdução à bioestatística. 3. ed. Rio de Janeiro: Campos, 1980.

Recebido em 07/06/2016

Aprovado em 03/08/2016 